In the usual use of asymptotic approximations it is a common practice to take $N$ such that $T_{N-1}$ is the smallest term in the asymptotic series. In many cases it is not clear which of two successive integers is to be taken as $N$, and this uncertainty is said to reflect an error comparable to $\left|T_{N-1}\right|$. Our (12a) and (13a) show that this earlier estimate may often be scarcely better than a crude guess and should be replaced by the present result.

A further complication can arise when term magnitude $\left|T_{N-1}\right|=\mid\left[c_{a}(N-1)\right]$ $x^{p_{0}+1-N} \mid$ passes through more than one relative minimum with respect to $N$. In such cases it may happen that the term minimum of smallest magnitude does not occur at the $N$ value giving the smallest error, $v_{a}(x)$. For instance, this arises in our example when $A_{1}=x=1, A_{3}=-\rho_{a}=0.3, A_{2}=4.2$ and $A_{4}=A_{5}=0$. In this case $\left|T_{1}\right|$ and $\left|T_{5}\right|$ are relative minima of $\left|T_{N-1}\right|$, and $\left|T_{1}\right|$ is about 20 times greater than $\left|T_{3}\right|$. But the error involved in taking $(N-1)=1$ is less than a third of that involved in taking $(N-1)=5$ and less than a sixth of $\left|T_{1}\right|$ itself.

One concludes, therefore, that for most accurate asymptotic approximation one must minimize the error, $v_{a}(x)$, with respect to the number of terms in $u_{a}(x)-N$-for each specific differential equation and for each chosen value of the independent variable, $x$. This can now be done for second order differential equations by using (12a) or (13a) and for higher order differential equations by using the more complicated result of Ref. [1].

\title{
APPEndix
}

As a brief statement of the result in Ref. [1], as it applies to (3), if we define

$$
\delta=\max _{\nu-1,2, \ldots, m}\left[\left(\text { degree of } q_{\nu}(\alpha, \rho) \text { in } \alpha \text { - degree of } q_{0}(\rho, \alpha) \text { in } \alpha\right) / \nu\right],
$$

if $\delta<1$ (which, if not already satisfied, can be brought about by substituting $x=z^{M}$ with $M$ a sufficiently large positive integer), and if $|\omega|=1$, then for any $N \geqq 2$

$$
x^{-\rho} v(x)=\sum_{\beta=N-1}^{\infty} \sum_{\mu=N-1}^{\beta} A_{\mu, \beta}(\omega)^{\beta-\mu}[c(\mu+1)] / \prod_{\mu=0}^{\beta}(x+\mu \omega),
$$

[where $A_{0, \beta}=A_{\mu, \mu-1}=0, A_{1,1}=0$, and, for $\beta>1$,

$$
\left.A_{\mu, \beta}=(\beta-1) A_{\mu,(\beta-1)}+A_{(\mu-1),(\beta-1)}\right]
$$

in $|x|>x_{0}$ and $|\arg x-\arg \omega| \leqq \pi / 2$. Incidentally, $\arg \omega$ is arbitrary.

\section{WAVE PROPAGATION IN A VISCO-ELASTIC MEDIUM*}

\section{By E. J. SCOTT (University of Illinois)}

1. Introduction. The use of mechanical models to describe the behavior of materials is well known [1]. By combining a Maxwell unit, consisting of a spring and dashpot connected in series, with a Voigt unit, consisting of a spring and dashpot connected in parallel, in various ways, it is possible to describe adequately in many cases the complex behavior of real materials. The stress-strain laws corresponding to a Voigt unit and a four parameter model consisting of a Maxwell unit combined in series with a Voigt

*Received Sept. 3, 1953. 
unit have been used to study the physical properties of high polymers and wave propagation in a visco-elastic medium [2].

It is the purpose of this article to consider the propagation of longitudinal waves in a medium confined between the two parallel planes $x=0$ and $x=l$ (Fig. 1) and whose

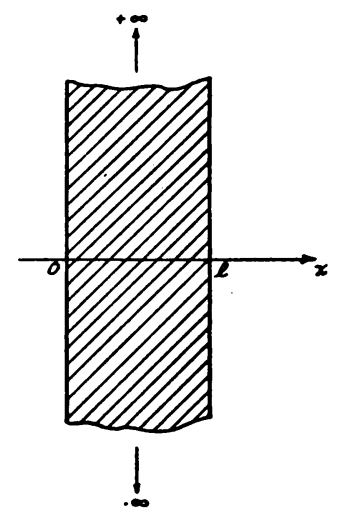

FIG. 1

physical behavior is representable by the linear mechanical model shown in Fig. 2 . The particular case of two Maxwell units coupled in parallel will be considered in detail because this model seems capable of describing some aspects of creep.

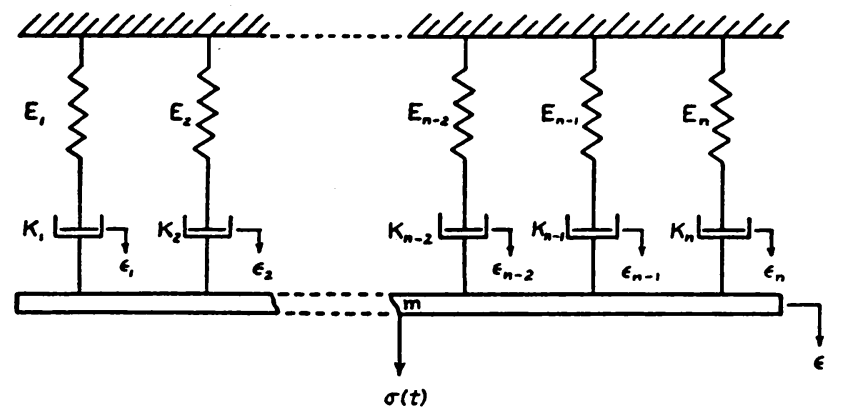

FIG. 2

2. Derivation of the partial differential equation. We shall first derive the stressstrain law that obtains for the linear model of Fig. 2. From elementary considerations, we have the following equations:

$$
\begin{gathered}
K_{i}\left[\epsilon_{t}(x, t)-\epsilon_{i t}(x, t)\right]=E_{i} \epsilon_{i}(x, t), \quad i=1,2, \cdots, n, \\
m \epsilon_{t \iota}(x, t)=\sigma(x, t)-\sum_{i=1}^{n} K_{i}\left[\epsilon_{t}(x, t)-\epsilon_{i \iota}(x, t)\right],
\end{gathered}
$$

where $x$ is a parameter and the subscripts involving $t$ denote differentiation with respect to time. By elimination, we obtain

$$
\sigma(x, t)=m \epsilon_{t t}(x, t)+\sum_{i=1}^{n} E_{i} \epsilon_{i}(x, t) .
$$


Solving Eq. (1), subject to the condition $\epsilon_{i}(x, 0)=0$, for $\epsilon_{i}(x, t)$ and substituting in (3), we find that the stress-strain law for the model under consideration is

$$
\sigma(x, t)=m \epsilon_{t \iota}(x, t)+\sum_{i=1}^{n} E_{i} \int_{0}^{t} \epsilon_{\tau}(x, \tau) \exp \left[-\mu_{i}(t-\tau)\right] d \tau,
$$

where $\mu_{i}=E_{i} / K_{i}$.

In the slab of thickness $l$ (Fig. 1), let $u(x, t)$ represent the displacement of the section $x$ at time $t$ and let $\rho$ be the density of the unstrained medium. Then

$$
\begin{aligned}
\sigma_{x}(x, t) & =\rho u_{\iota t}(x, t), \\
\epsilon(x, t) & =u_{x}(x, t),
\end{aligned}
$$

where the subscripts denote partial differentiation with respect to the corresponding variable.

Eliminating $\sigma$ and $\epsilon$ we obtain the following integro-differential equation for the propagation of longitudinal waves in the medium under consideration

$$
\rho u_{t t}(x, t)=m u_{x x t t}(x, t)+\sum_{i=1}^{n} E_{i} \int_{0}^{t} u_{x x \tau}(x, \tau) \exp \left[-\mu_{i}(t-\tau)\right] d \tau .
$$

3. Solution. In what follows we shall assume that the material is initially unstrained and at rest, so that

$$
u(x, 0)=u_{t}(x, 0)=0 . \quad(0 \leq x \leq l) .
$$

Applying the Laplace transform

$$
u^{*}(x, p)=L_{t}\{u(x, t)\}=\int_{0}^{\infty} e^{-p t} u(x, t) d t
$$

to Eq. (7) and making use of conditions (8), we obtain

$$
\rho p u^{*}(x, p)=m p \frac{d^{2} u^{*}(x, p)}{d x^{2}}+\frac{d^{2} u^{*}(x, p)}{d x^{2}} \sum_{i=1}^{n} \frac{E_{i}}{p+\mu_{i}},
$$

from which

$$
\frac{d^{2} u^{*}(x, p)}{d x^{2}}-\frac{\rho p u^{*}(x, p)}{m p+\sum_{i=1}^{n} E_{i} /\left(p+\mu_{i}\right)}=0 .
$$

The solution of this equation suitable for the finite region being considered is

$$
u^{*}(x, p)=c_{1} \cosh x Z_{n}^{1 / 2}+c_{2} \sinh x Z_{n}^{1 / 2},
$$

where $Z_{n}=\rho p /\left[m p+\sum_{i=1}^{n} E_{i} /\left(p+\mu_{i}\right)\right]$.

Let us now suppose that the section $x=l$ is fixed and the section $x=0$ is given an impulsive velocity $V_{0}$ which is then maintained, i.e., let

$$
u_{t}(0, t)=V_{0}, \quad u(l, t)=0, \quad(t>0),
$$

whence

$$
u^{*}(0, p)=V_{0} / p^{2} \quad u^{*}(l, p)=0 .
$$


These conditions when applied to Eq. (10) yield the equations

$$
V_{0} / p^{2}=c_{1}, \quad 0=c_{1} \cosh l Z^{1 / 2}+c_{2} \sinh l Z^{1 / 2} .
$$

The values of $c_{1}$ and $c_{2}$ obtained from Eqs. (13), when they are substituted in Eq. (10) and the result is simplified, give

$$
u^{*}(x, p)=\frac{V_{0} \sinh (l-x) Z_{n}^{1 / 2}}{p^{2} \sinh l Z_{n}^{1 / 2}}
$$

whence, by the complex inversion theorem,

$$
u(x, t)=\frac{V_{0}}{2 \pi i} \int_{c-i \infty}^{c+i \infty} e^{p t} \frac{\sinh (l-x) Z_{n}^{1 / 2}}{p^{2} \sinh l Z_{n}^{1 / 2}} d p .
$$

4. A special case. We shall consider the solution, in detail, for the special case of a model for which the number of Maxwell units coupled in parallel is two and $m=0$. In this instance Eq. (15) becomes

$$
u(x, t)=\frac{V_{0}}{2 \pi i} \int_{c-i \infty}^{c+i \infty} e^{p t} \frac{\sinh (l-x) Z_{2}^{1 / 2}}{p^{2} \sinh l Z_{2}^{1 / 2}} d p,
$$

where $Z_{2}=\rho p\left(p+\mu_{1}\right)\left(p+\mu_{2}\right) /\left[\left(E_{1}+E_{2}\right) p+E_{1} \mu_{2}+E_{2} \mu_{1}\right]$.

The integrand is a single-valued function of $p$ with a double pole at $p=0$ and poles at those values of $p$ which satisfy the cubic equation

$$
p^{3}+\sigma_{1} p^{2}+\left(\frac{n^{2} \pi^{2}}{l^{2} \rho} \sigma_{3}+\sigma_{2}^{2}\right) p+\frac{n^{2} \pi^{2}}{l^{2} \rho} \sigma_{4}^{2}=0, \quad n=1,2,3, \cdots,
$$

where $\sigma_{1}=\mu_{1}+\mu_{2}, \sigma_{2}^{2}=\mu_{1} \mu_{2}, \sigma_{3}=E_{1}+E_{2}, \sigma_{4}^{2}=E_{1} \mu_{2}+E_{2} \mu_{1}$. Since the coefficients of Eq. (17) are all positive, its roots, if they are all real, will all be negative. Furthermore, since

$$
\sigma_{1}\left(\frac{n^{2} \pi^{2}}{l^{2} \rho} \sigma_{3}+\sigma_{2}^{2}\right)>\frac{n^{2} \pi^{2}}{l^{2} \rho} \sigma_{4}^{2}, \quad n=1,2,3, \cdots
$$

if Eq. (17) has one negative and two conjugate complex roots, the real parts of the complex roots are negative [3].

Making the substitution $p=y-\sigma_{1} / 3$ in Eq. (17), we find that

$$
\begin{aligned}
y^{3}+\left(\frac{n^{2} \pi^{2}}{l^{2} \rho} \sigma_{3}\right. & \left.+\sigma_{2}^{2}-\sigma_{1}^{2} / 3\right) y \\
& +\left[\left(\sigma_{4}^{2}-\sigma_{1} \sigma_{3} / 3\right)+\frac{2}{27} \sigma_{1}^{3}-\sigma_{1} \sigma_{2}^{2} / 3\right]=0, \quad n=1,2,3, \cdots .
\end{aligned}
$$

Case I. If $n^{2} \pi^{2} \sigma_{3} /\left(l^{2} \rho\right)+\sigma_{2}^{2}>\sigma_{1}^{2} / 3, n=1,2,3, \cdots$, then the coefficient of $y$ in Eq. (18) is positive and Eq. (18) has one real root and two complex roots. Consequently the roots of Eq. (17) can be represented thus

$$
p=-a_{n}, \quad p=-b_{n}+i c_{n}, \quad p=-b_{n}-i c_{n},
$$

where $a_{n}>0, b_{n}>0$ and $n=1,2,3, \cdots$. 
The residue of the integrand of Eq. (16) at the double pole $p=0$ is

$$
\frac{l-x}{l} t+\frac{\rho \sigma_{2}^{2} x(l-x)(x-2 l)}{6 l \sigma_{4}^{2}},
$$

and that at $p=-a_{n}$ is

$$
\left.\frac{e^{\nu t} \sinh (l-x) Z_{2}^{1 / 2}}{p^{2} d\left(\sinh l Z_{2}^{1 / 2}\right) / d p}\right]_{p=-a_{n}}=(-1)^{n+1} \frac{2 n \pi\left(\sigma_{4}^{2}-\sigma_{3} a_{n}\right) e^{-a_{n} t} \sin [n \pi(l-x) / l]}{a_{n}^{2} l^{2}\left[\rho\left(3 a_{n}^{2}-2 \sigma_{1} a_{n}+\sigma_{2}^{2}\right)+\sigma_{3} n^{2} \pi^{2} / l^{2}\right]} .
$$

Furthermore, the residue at $p=-b_{n}+i c_{n}$ is

$$
\begin{aligned}
& \left.\frac{e^{\nu t} \sinh (l-x) Z_{2}^{1 / 2}}{p^{2} d\left(\sinh l Z_{2}^{1 / 2}\right) / d p}\right]_{p=-b_{n}+i c_{n}} \\
& \quad=(-1)^{n+1} \frac{2 n \pi\left[\left(A_{n} C_{n}+B_{n} D_{n}\right)+i\left(A_{n} D_{n}-B_{n} C_{n}\right)\right] \sin [n \pi(l-x) / l] \exp \left(-b_{n}+i c_{n}\right) t}{l^{2}\left(A_{n}^{2}+B_{n}^{2}\right)\left[\left(b_{n}^{2}-c_{n}^{2}\right)^{2}+4 b_{n}^{2} c_{n}^{2}\right]}
\end{aligned}
$$

and that at $p=-b_{n}-i c_{n}$ is

$$
(-1)^{n+1} \frac{2 n \pi\left[\left(A_{n} C_{n}+B_{n} D_{n}\right)-i\left(A_{n} D_{n}-B_{n} C_{n}\right)\right] \sin [n \pi(l-x) / l] \exp \left(-b_{n}-i c_{n}\right) t}{l^{2}\left(A_{n}^{2}+B_{n}^{2}\right)\left[\left(b_{n}^{2}-c_{n}^{2}\right)^{2}+4 b_{n}^{2} c_{n}^{2}\right]},
$$

where

$$
\begin{aligned}
& A_{n}=3 \rho\left(b_{n}^{2}-c_{n}^{2}\right)-2 \sigma_{1} \rho b_{n}+\rho \sigma_{2}^{2}-\sigma_{3} n^{2} \pi^{2} / l^{2}, \\
& B_{n}=2 \rho c_{n}\left(\sigma_{1}-b_{n}\right) \\
& C_{n}=\sigma_{4}^{2} b_{n}^{2}-b_{n}^{3} \sigma_{3}-\sigma_{4}^{2} c_{n}^{2}-b_{n} c_{n}^{2} \sigma_{3}, \\
& D_{n}=2 b_{n} c_{n} \sigma_{4}^{2}-\sigma_{3} c_{n}^{3}-b_{n}^{2} c_{n} \sigma_{3} .
\end{aligned}
$$

Therefore,

$$
\begin{aligned}
u(x, t)= & V_{0}\left\{(l-x) t / l+\rho \sigma_{2}^{2} x(l-x)(x-2 l) /\left(6 l \sigma_{4}^{2}\right)\right. \\
& +\frac{2 \pi}{l^{2}} \sum_{n=1}^{\infty}(-1)^{n+1} \frac{n\left(\sigma_{4}^{2}-\sigma_{3} a_{n}\right) \sin [n \pi(l-x) / l] e^{-a_{n} \ell}}{a_{n}^{2}\left[\rho\left(3 a_{n}^{2}-2 \sigma_{1} a_{n}+\sigma_{2}^{2}\right)+\sigma_{3} n^{2} \pi^{2} / l^{2}\right]} \\
& \left.+\frac{4 \pi}{l^{2}} \sum_{n=1}^{\infty}(-1)^{n+1} \frac{n\left(M_{n}^{2}+N_{n}^{2}\right)^{1 / 2} e^{-b_{n} t} \cos \left(c_{n} t-\phi_{n}\right) \sin [n \pi(l-x) / l]}{\left(A_{n}^{2}+B_{n}^{2}\right)\left[\left(b_{n}^{2}-c_{n}^{2}\right)^{2}+4 b_{n}^{2} c_{n}^{2}\right]}\right\},
\end{aligned}
$$

where $M_{n}=A_{n} C_{n}+B_{n} D_{n}, N_{n}=A_{n} D_{n}-B_{n} C_{n}$ and $\tan \phi_{n}=N_{n} / M_{n}$.

Case II. Suppose that $n^{2} \pi^{2} \sigma_{3} /\left(l^{2} \rho\right)+\sigma_{2}^{2}<\sigma_{1}^{2} / 3$ for $n=1,2, \cdots, k$, where $k$ is the largest integer for which the inequality holds. Then Eq. (18) may have three real roots and Eq. (17) three real negative roots. For $n=k+1, k+2, \cdots$, however, $n^{2} \pi^{2} \sigma_{3} /\left(l^{2} \rho\right)+\sigma_{2}^{2}>\sigma_{1}^{2} / 3$ and the solution will have the terms given in Eq. (19). Therefore, if the first inequality mentioned above obtains for $n<k$ and the second for $n \geq k$, then the solution will contain the terms displayed in Eq. (19) plus (possibly) three summations of terms like those occurring in the first summation of Eq. (19).

An examination of the solution reveals that as $t \rightarrow \infty$ the transient terms following the summation signs become negligible and the oscillations tend to a state characterized by the first two terms of Eq. (19). 
5. Discussion of a numerical example. Figures 3 and 4 show the piots of $u\left\{x, t, V_{0}\right.$ and $u_{t}(x, t) / V_{0}$ versus $t$, respectively, for different cross-sectional values $x$ of a medium

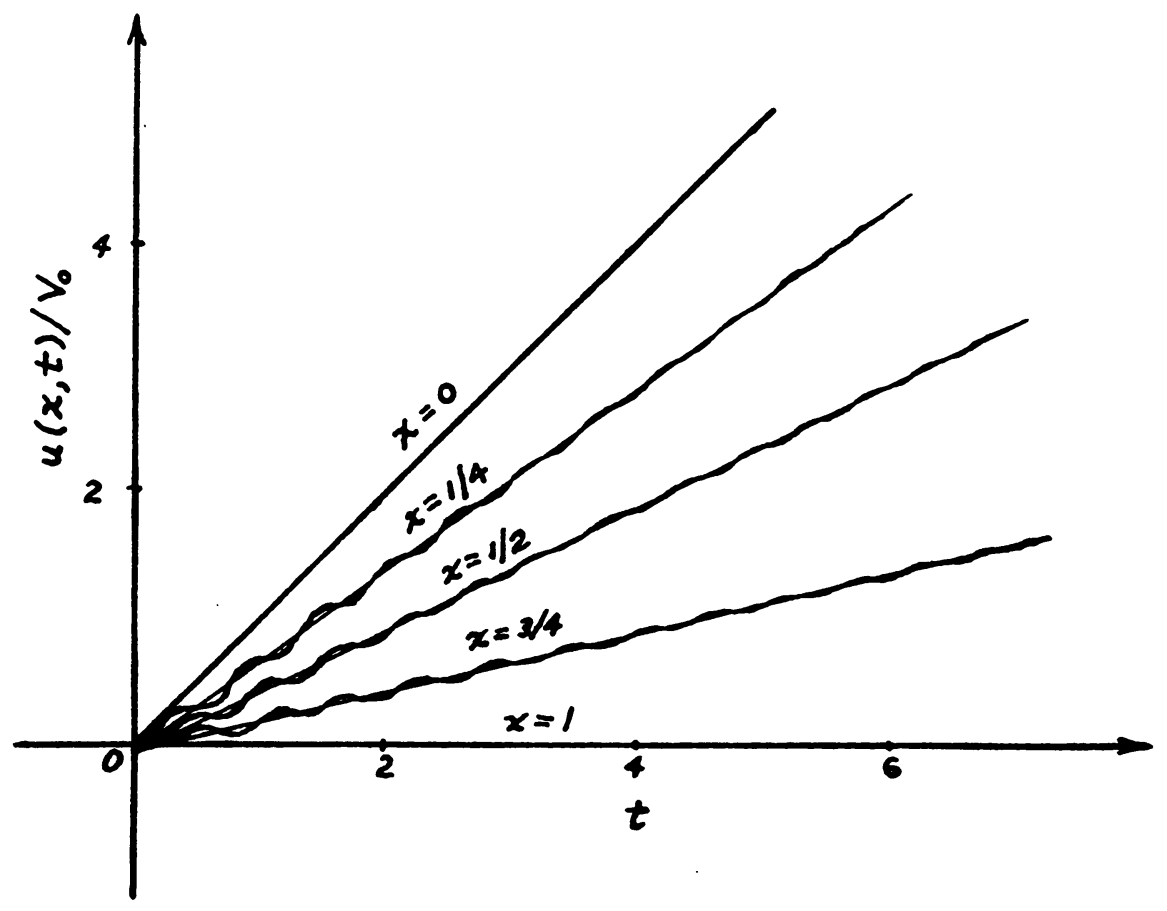

Fra. 3

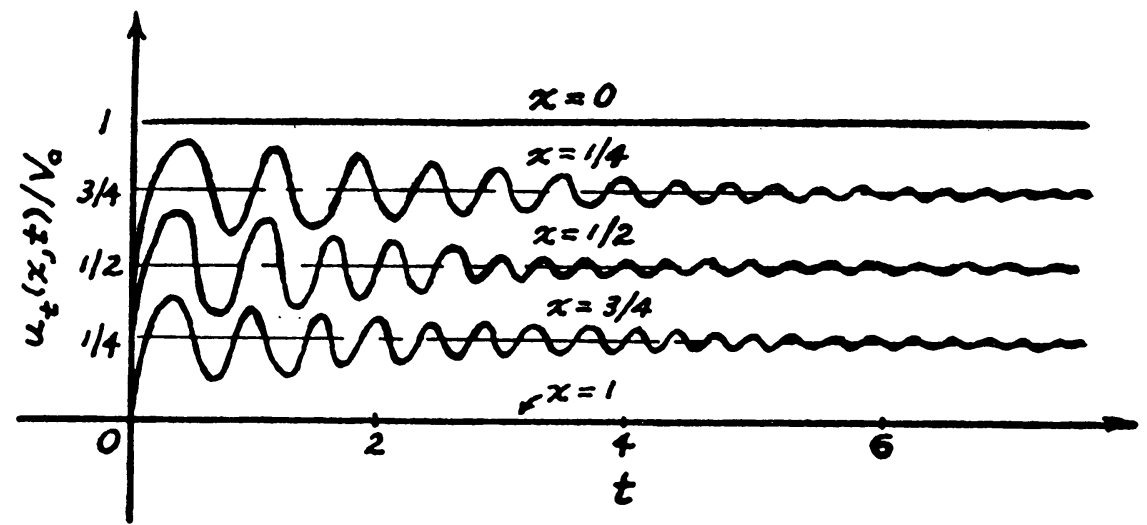

FIG. 4

characterized by two Maxwell units coupled in parallel and such that $m=0, E_{1}=E_{2}=$ $K_{1}=K_{2}=\rho=l=1$. Both graphs indicate the damped oscillatory character of the displacement as well as the velocity of the cross-sections between $x=0$ and $x=1$ of the medium. As $t \rightarrow \infty$ the oscillations die out and the displacement of a section increases uniformly while its velocity becomes constant. While the graphs depict the general character of the motion, computations were carried out for a limited number of points and exact values are not implied at all points. 


\section{BIBLIOGRAPHY}

1. T. Alfrey, Mechanical behavior of high polymers, Interscience Pub., New York, 1948.

2. ' D. Glauz, The visco-elastic vibrating reed, Technical Report No. 4, PA-TR-4/22, and Transient ave analysis in linear time dependent material by the same author, Technical Report No. 2, P'A-TR-2/25, Graduate Division of Applied Mathematics, Brown Unjversity, Providence, R. I.

3. J. P. Den Hartog, Mechanical vibrations, McGraw-Hill Book Co., Inc., New York, 1947.

\section{NOTE ON TAYLOR INSTABILITY*}

\section{BY GARRETT BIRKHOFF (Harvard University)}

1. Qualitative discussion. In a well-known paper [3], Sir Geoffrey Taylor has discussed the stability under normal acceleration of a plane interface separating two fluids of different density. His main conclusion (reached several years earlier) is now classic: the interface is unstable when the light fluid is accelerated towards the dense fluid and (presumably) stable when the reverse holds. This conclusion has important applications to gas-filled underwater explosion bubbles.

Its applicability to small vapor-filled cavities is however less clear. The stabilizing role of surface tension is known ${ }^{1}$ to be important, and Binnie [1] has suggested that surface tension may even be sufficient to compensate for Taylor instability.

The purpose of this note is to show that, in spite of the fact that the denser liquid is being accelerated towards the lighter vapor, collapsing bubbles are unstable, and that this result is unaffected by surface tension (though it may be affected by viscosity or thermodynamic considerations). The proof of this fact depends on a consideration of the stability of differential equations near regular singular points: the instability is algebraic, and not of the exponential type usually considered.

2. Negative damping. The formulas underlying the perturbation theory of collapsing spherical cavities are easily found ${ }^{2}$. Let the cavity radius $b(t)$ be given as a function of time, and the interface expressed in spherical coordinates in terms of Legendre polynomials by

$$
r=b(t)+\sum_{h=1}^{\infty} b_{h}(t) P_{h}(\cos \phi) .
$$

Supposing the $b_{h}(t)$ small, and neglecting gravity and surface tension, the condition of constant internal pressure gives $\dagger$

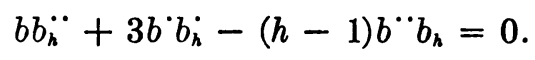

The same formula applies to any surface harmonic of order $h$.

${ }^{*}$ Received Oct. 19, 1953.

${ }^{1}$ This was observed independently in 1951 by R. H. Pennington and R. Bellman at Princeton, and by $R$. L. Ingraham and the author, but not published.

${ }^{2}$ Formulas (1)-(2) were derived by W. G. Penney and A. T. Price, British Report SW-27 (1942); see R. H. Cole, Underwater explosions, Princeton, 1948, p. 311. See also [1, Formulas (2)-(3)].

$\dagger$ For typographical reasons, the dots indicating time derivatives are set as superscripts. 